\title{
Theory of cyclotron resonance in a cylindrical non-neutral plasma
}

Roy W. Gould

Applied Physics, California Institute of Technology, Pasadena, California 91125

(Received 28 November 1994; accepted 22 December 1994)

\begin{abstract}
Resonances near the cyclotron frequency of a cylindrical non-neutral plasma column with radial density and angular velocity profiles are studied. Cold plasma and warm plasma models are used to consider the various multipole modes $\left(e^{i m \theta}\right)$ for $k_{z}=0$. For $m=1$ only a single resonance, a center of mass mode, is found. Its frequency is downshifted from the cyclotron frequency by an amount equal to the frequency of the low-frequency diocotron mode. For each $m$ greater than 1 the cold plasma model gives a continuous absorption band with spatially localized absorption at each frequency, corresponding to a continuum of singular modes. In the warm plasma model, the continuuo go over to sets of discrete radially trapped azimuthally propagating Bernstein modes. For both cases, the plasma rotation Doppler-shifts the azimuthally propagating modes up in frequency. The angular velocity profile, together with plasma temperature, determine the spacing of the Bernstein modes within a band. (C) 1995 American Institute of Physics.
\end{abstract}

\section{INTRODUCTION}

In this paper the modes of a cylindrical non-neutral plasma column in the vicinity of the cyclotron frequency $\omega_{c}=e B / m_{e}$ are studied theoretically. This work was done in support of recent experiments. ${ }^{1}$ Plasma rotation plays a vital role in determining the mode structure near the cyclotron frequency of non-neutral plasmas and is responsible for marked differences from otherwise similar phenomena in neutral plasmas. Non-neutral plasmas have been studied extensively in recent years and there are two good summaries of this work. ${ }^{2,3}$ However there has been little attention to high frequency phenomena. A notable exception is the early theoretical work of Krall and Davidson ${ }^{3,4}$ in which they show how to carry over certain Vlasov results from cylindrical neutral plasmas to cylindrical non-neutral plasmas. However, their results are limited to the rigid rotor equilibrium state, a situation not always obtained in laboratory plasmas because of the time required to reach this state can exceed the plasma lifetime. Recently Dubin ${ }^{5}$ has given a theory of the modes of cold rigid rotor spheroidal non-neutral plasma, which includes high frequency modes. Pearson ${ }^{6}$ gave a finite Larmor radius treatment of the Bernstein ${ }^{7}$ waves of a slab and cylindrical neutral plasmas with a spatially varying density profile but rotation was not included in the cylindrical case. More recently Prasad, Morales, and Fried ${ }^{8}$ have have given a small Larmor radius theory of modes of a nonneutral slab plasma which has sheared flow. However, because of the slab geometry, their results cannot be compared directly with experiments.

In this paper, we are particularly interested in the modes of a nonequilibrium plasma in which both plasma density and angular velocity are functions of radius. While these profiles are expected to evolve toward thermal equilibrium, this evolution takes place on a sufficiently long time scale that the profiles may be considered to be in a steady state for the purposes of describing the phenomena discussed here. For simplicity only the $k_{z}=0$ modes of a long column with the various cylindrical harmonics, $m \neq 0$, are treated. The cold plasma case, which leads to a continuous spectrum of modes for cach $m$, is discussed first. Then an approximate treatment of the warm plasma modes (first order in temperature) is given. This leads to azimuthally propagating and radially trapped Bernstein modes, reminiscent of the Buchsbaum-Hasegawa modes ${ }^{9}$ which occur near $2 \omega_{c}$ in a neutral plasma which has a radial density profile but no rotation. ${ }^{10}$

It is instructive as a preliminary to recall the behavior of a cold cylindrical neutral plasma whose density depends upon radius but which is not rotating. When an oscillating electric field is applied externally, the plasma exhibits a spatially localized resonant response at the radius where $K_{\perp}(r)=1-\omega_{p}^{2}(r) /\left(\omega^{2}-\omega_{c}^{2}\right)$, the perpendicular dielectric function, vanishes. Because of the radial dependence of density, there is a range of frequencies for which this condition can be satisfied, corresponding to the range of upper hybrid frequencies within the plasma. This leads to a continuum of singular eigenmodes, similar to those found in other problems, ${ }^{1 \mathrm{f}, 12}$ and an upper-hybrid continuum absorption band.

A non-neutral plasma column is necessarily in rotation in order to be in a steady state. This rotation gives rise to a Doppler shift of an applied frequency, and the upper hybrid resonance, as seen in the laboratory frame is upshifted. As will be seen later, the centrifugal and Coriolis forces associated with the rotation also give rise to a downshift of the single particle gyration frequency. For rigid rotor equilibria the downshifted frequency is called the vortex frequency. ${ }^{3}$ For nonrigid rotor steady states, the single particle downshift and the Doppler upshift both depend upon radius and this complicates the situation considerably.

In the remainder of the paper the response of the plasma to applied multipolar fields produced by the geometry of Fig. $I$ is studied. In Secs. II-VI the cold plasma behavior of electrons and the absorption band which arises out of the continuum of singular normal modes is discussed. In Sec. VII it is shown, approximately, how the continuum goes over to a set of radially trapped azimuthally propagating Bernstein modes in a warm plasma, where finite Larmor radius effects are important. In Sec. VIII the results are extended to mul- 


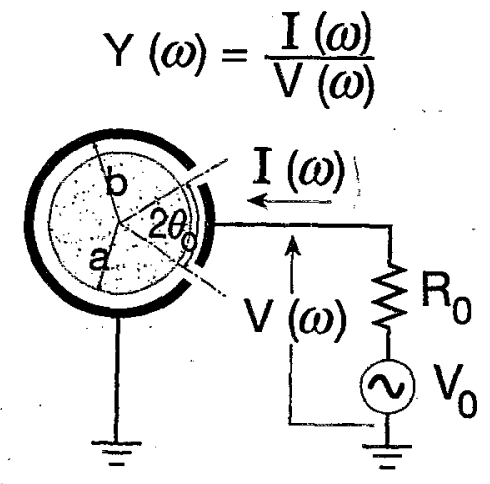

FIG. 1. Schematic diagram of the plasma, surrounding electrodes, and driving circuitry (end view), with definition of $Y(\omega)$, the plasma admittance function with $\omega=\omega_{c}+\omega_{0}(0) \lambda$.

tiple ion species. Throughout the paper it is assumed that $\omega_{p}^{2} / \omega_{c}^{2}$ is a small parameter, and results are obtained only to first order in this parameter. It is also assumed that the dimensions are small compared with a free space wavelength so that retardation effects can be neglected.

\section{COLD PLASMA EQUATIONS}

We consider a cylindrical non-neutral plasma which is contained by a static axial magnetic field $B_{0}$ and which has a radial density profile $n_{0}(r)$ which is assumed to be known. The density profile, $n_{0}(r)$, need not be an equilibrium profile in the thermodynamic sense, since a variety of nonequilibrium profiles can be produced which evolve on a time scale very long compared to the time scale of cyclotron resonance observation times. Associated with a given density profile, $n_{0}(r)$, is a radial electric field profile, $E_{0}(r)$, and an angular velocity profile, $\omega_{0}(r)$, which is described in Sec. III.

The cold fluid equations of motion and continuity in Eulerian form for cylindrical geometry and no $z$ variation are

$$
\begin{aligned}
& \frac{\partial u_{r}}{\partial t}+\frac{u_{r} \partial u_{r}}{\partial r}+\left(\frac{u_{\theta}}{r}\right) \frac{\partial u_{r}}{\partial \theta}-\frac{u_{\theta}^{2}}{r}=-\left(\frac{e}{m_{e}}\right)\left[E_{r}+u_{\theta} \ddot{B}_{0}\right] \\
& \frac{\partial u_{\theta}}{\partial t}+\frac{u_{r} \partial u_{\theta}}{\partial r}+\left(\frac{u_{\theta}}{r}\right) \frac{\partial u_{\theta}}{\partial \theta}+\frac{u_{\theta} u_{r}}{r}=-\left(\frac{e}{m_{e}}\right)\left[E_{\theta}-\dot{u}_{r} B_{0}\right], \\
& \frac{\partial n}{\partial t}+\left(\frac{1}{r}\right) \frac{\partial\left(r n u_{r}\right)}{\partial r}+\left(\frac{1}{r}\right) \frac{\partial\left(n u_{\theta}\right)}{\partial \theta}=0 .
\end{aligned}
$$

Maxwell equations for the quasistatic electric field are

$$
\begin{aligned}
& \left(\frac{1}{r}\right) \frac{\partial\left(r E_{\theta}\right)}{\partial r}-\left(\frac{1}{r}\right) \frac{\partial E_{r}}{\partial \theta}=0, \\
& \left(\frac{1}{r}\right) \frac{\partial\left(r E_{r}\right)}{\partial r}+\left(\frac{1}{r}\right) \frac{\partial E_{\theta}}{\partial \theta}=-\frac{n e}{\epsilon_{0}} .
\end{aligned}
$$

\section{THE STEADY STATE}

In the steady state, $E_{\theta}$ and $u_{r}$ vanish and the remaining quantities, $E_{r}, u_{\theta}$, and $n$ are independent of $\theta$ and $t$. The steady-state quantities obey the equations

$$
\begin{aligned}
& r \omega_{0}^{2}=\left(\frac{e}{m_{e}}\right) E_{0 r}+r \omega_{c} \omega_{0}, \\
& \frac{d\left(r E_{0 r}\right)}{d r}=-\frac{r n_{0} e}{\epsilon_{0}}
\end{aligned}
$$

where $\omega_{0}(r)=u_{0 \theta}(r) / r$ is the angular velocity at radius $r$. The subscripts 0 refer to the steady-state values. In the low density limit, $\left(\omega_{p}^{2} / \omega_{c}^{2}\right) \ll 1$, the rotational angular velocity, $\omega_{0}$, is everywhere small compared with the cyclotron frequency and the centrifugal force term $r \omega_{0}^{2}$ of Eq. (6) may be neglected. This corresponds to being well below the Brillouin density limit. Equations (6) and (7) then lead to the usual low-density relationship between angular velocity and density:

$$
\left(\frac{1}{r}\right) \frac{d\left[r^{2} \omega_{0}(r)\right]}{d r}=\frac{\omega_{p}^{2}(r)}{\omega_{c}} .
$$

It is convenient to normalize the radial-dependent plasma frequency and rotational angular velocity to their central values and introduce the functions $f(r)$ and $g(r)$ to express the normalized density and angular velocity functions. Let

$$
\begin{aligned}
& \omega_{p}^{2}(r)=\omega_{p}^{2}(0) f(r), \\
& \omega_{0}(r)=\omega_{0}(0) g(r),
\end{aligned}
$$

where, by definition, $f(0)=g(0)=1$. It follows from Eq. (8) that the central rotation frequency is $\omega_{0}(0)=\omega_{p}^{2}(0) / 2 \omega_{c}$. With these definitions Eq. (8) takes the form

$$
\frac{d\left[r^{2} g(r)\right]}{d r}=2 r f(r) \text {. }
$$

A simple illustration of a monotonically "decreasing density profile is the parabolic density profile $f(r)=1-(r / a)^{2}$, for $r<\dot{a}$. This profile, together with the corresponding angular velocity profile, $g(r)=1-0.5(r / a)^{2}$ will be uscd in later discussions, although the general approach is not limited to this example; $g(r)$ is obtained by integrating Eq. $\left(8^{\prime}\right)$ and both $f(r)$ and $g(r)$ are shown in Fig. 2.

\section{SMALL PERTURBATIONS}

The linearized forms of Eqs. (1)-(3) for small perturbations (subscript 1) when one assumes $\exp (i m \theta-i \omega \hat{t})$ dependence are

$$
\begin{aligned}
& -i\left[\omega-m \omega_{0}\right] u_{1, r}+\left[\omega_{c}-2 \omega_{0}\right] u_{1 \theta}=-\left(\frac{e}{m_{e}}\right) E_{1 r} \\
& -\left[\omega_{c}-2 \omega_{0}-\frac{r d \omega_{0}}{d r}\right] u_{1 r}-i\left[\omega-m \omega_{0}\right] u_{1 \theta}=-\left(\frac{e}{m_{e}}\right) E_{1 \theta}, \\
& -i\left(\omega-m \omega_{0}\right) n_{1}+\left(\frac{1}{r}\right) \frac{d\left(r n_{0} u_{1 r}\right)}{d r}+\left(\frac{i m}{r}\right) n_{0} u_{1 \theta}=0
\end{aligned}
$$

which are readily solved for $u_{1 r}$ and $u_{1 \theta}$ in terms of $E_{1 r}$ and $E_{1 \theta}$ : 


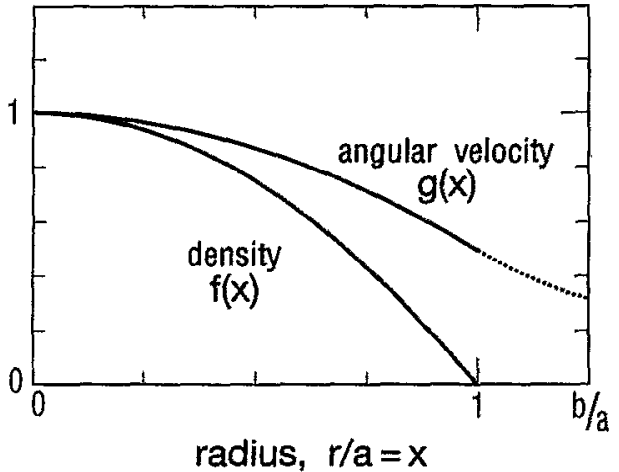

FIG. 2. Illustrative density and angular velocity profiles of a rotating nonneutral plasma, normalized to their central values. $f(x)=n_{0}(x) / n_{0}(0)$, $g(x)=\omega_{0}(x) / \omega_{0}(0), x=r / a$.

$$
\begin{aligned}
u_{1 r}= & -\left(\frac{e}{m_{e}}\right) \\
& \times \frac{-i\left[\omega-m \omega_{0}\right] E_{1 r}-\left[\omega_{c}-2 \omega_{0}\right] E_{1 \theta}}{-\left[\omega-m \omega_{0}\right]^{2}+\left[\omega_{c}-2 \omega_{0}\right]\left[\omega_{c}-2 \omega_{0}-r d \omega_{0} / d r\right]},
\end{aligned}
$$

$$
\begin{aligned}
u_{1 \theta}= & -\left(\frac{e}{m_{e}}\right) \\
& \times \frac{\left[\omega_{c}-2 \omega_{0}-r d \omega_{0} / d r\right] E_{1,}-i\left[\omega-m \omega_{0}\right] E_{1 \theta}}{-\left[\omega-m \omega_{0}\right]^{2}+\left[\omega_{c}-2 \omega_{0}\right]\left[\omega_{c}-2 \omega_{0}-r d \omega_{0} / d r\right]} .
\end{aligned}
$$

Now $\omega^{\prime}=\omega-m \omega_{0}(r)$ is the local Doppler shifted frequency seen by a fluid element drifting around the axis at radius $r$. The other term in the denominator of Eqs. (14) and (15) can be identified as the square of the effective single particle gyration frequency

$$
\omega_{c}^{\prime}=\left[\left(\omega_{c}-2 \omega_{0}\right)\left(\omega_{c}-2 \omega_{0}-\frac{r d \omega_{0}}{d r}\right)\right]^{1 / 2} .
$$

This frequency is downshifted slightly from $\omega_{c}=e B_{0} / m_{e}$ by the centrifugal and Coriolis forces and, in general, the downshift is also dependent upon radius. However, in the special case when the plasma density is independent of radius, $\omega_{0}$ is also independent of radius and one recovers the rigid rotor vortex frequency, $\omega_{c}^{\prime}=\omega_{c}-2 \omega_{0}$, which is also independent of radius.

Since we are primarily interested in frequencies close to the cyclotron frequency Eqs. (14) and (15) can be simplified. It is useful to introduce the normalized frequency $\lambda$ through the definition

$$
\omega=\omega_{c}+\left[\omega_{p}^{2}(0) / 2 \omega_{c}\right] \lambda,
$$

where $\lambda$ is the frequency difference $\omega-\omega_{c}$, normalized to the central rotation frequency. Then to first order in $\omega_{p}^{2}(0) / \omega_{c}^{2}$, Eqs. (14) and (15) can be written

$$
n_{0} e u_{1 r}=-i n_{0} e u_{1 \theta}=\frac{-i \omega_{c} \epsilon_{0} f(r)\left[E_{1 r}-i E_{i \theta}\right]}{[\lambda-m g+2 g+0.5 r d g / d r]} \text {, }
$$

or, more simply, as $n_{0} e u_{+}=0$ and

$$
n_{0} e u_{-}=\frac{-2 i \omega_{c} \epsilon_{0} f(r) E_{-}}{[\lambda-m g+g+f]},
$$

where the definitions $E_{ \pm}=E_{1 r} \pm i E_{1 \theta}$ and $u_{ \pm}=u_{1 r} \pm i u_{1 \theta}$ have been introduced, and $d g / d r$ from Eq. $\left(8^{\prime}\right)$ has been used. $E_{ \pm}$and $u_{ \pm}$denote amplitudes of the two circularly polarized components of the electric field and the velocity. From this result it is seen that close to cyclotron resonance only one circularly polarized component is effective in exciting the motion, namely that which matches the gyration sense of the particles. The velocity which it produces is circularly polarized with the same sense. Linear combinations of the two Maxwell equations, Eqs. (4) and (5) give

$$
\left(\frac{1}{r}\right) \frac{d\left(r E_{ \pm}\right)}{d r} \pm\left(\frac{m}{r}\right) E_{ \pm}=\frac{-n_{1} e}{\epsilon_{0}}
$$

and the continuity equation, Eq. (13), together with Eq. (18) gives

$$
-n_{1} e / \epsilon_{0}=\frac{1}{r} \frac{d}{d r} \frac{r f E_{-}}{[\lambda-m g+g+f]}-\frac{m}{r} \frac{f E_{-}}{[\lambda-m g+g+f]},
$$

where $\omega-m \omega_{0} \approx \omega_{c}$ has been used. Defining a dielectric function for right circularly polarized fields as $K(r)=1-f l$ $[\lambda-m g+g+f]$, the quantity $D_{-}=K E_{-}$obeys a particularly simple equation:

$$
\frac{1}{r} \frac{d}{d r}\left(r D_{-}\right)-\frac{m}{r} D_{-}=0 ;
$$

$D_{-}$is the displacement associated with the resonantly polarized component.

\section{COLD PLASMA SOLUTIONS}

The solution of Eq. (22) is simply $D_{-}(r)=A r^{m-1}$, where $A$ is an arbitrary constant, and the corresponding electric field is

$$
E_{-}(r)=A r^{m-1} / K(r) .
$$

For $m \leqslant 0, A$ must be zero in order for the field to be zero at

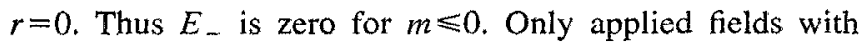
positive $m$ have the correct circular polarization to elicit a response from a pure electron plasma. Thus only positive $m$ need be considered. For $m=+1, D_{-}=A$ is independent of radius. This corresponds to a center of mass mode.

Having obtained the solution for $E_{-}$, it is substituted into Eq. (21) to obtain the perturbed density, and the latter is then substituted into Eq. (20) to obtain the equation for $E_{+}$:

$$
\begin{aligned}
\frac{1}{r} \frac{d}{d r}\left(r E_{+}\right)+\frac{m}{r} E_{+}= & \frac{1}{r} \frac{d}{d r}\left\{r A r^{m-1}\left[1-\frac{1}{K}\right]\right\} \\
& -\frac{m}{r} A r^{m-1}\left[1-\frac{1}{K}\right] .
\end{aligned}
$$

The right side of Eq. (24) gives the perturbed density in the plasma and this expression can be used to obtain the radial dependence of the perturbed density for various applied frequencies. The perturbed density exhibits a singularity at the 
radius where $K(r)$ vanishes. This condition corresponds to a localized upper hybrid resonance condition, when the various frequency shifts incorporated into $K(r)$ are taken into account. To solve for $E_{+}$, the substitution $E_{+}=\psi(r) / r^{(m+1)}$ leads to an integrable equation for $\psi$, whose solution is

$$
\psi(r)=A \int_{0}^{r} \frac{d}{d r^{\prime}}\left[\frac{1}{K\left(r^{\prime}\right)}-1\right] r^{\prime 2 m} d r^{\prime}
$$

The ratio of $E_{+}$to $E_{-}$at a given radius is then given by

$$
R_{m}(r)=\frac{E_{+}(r)}{E_{-}(r)}=\frac{K(r)}{r^{2 m}} \int_{0}^{r} \frac{d}{d r^{\prime}}\left[\frac{1}{K\left(r^{\prime}\right)}-1\right] r^{\prime 2 m} d r^{\prime} .
$$

At a radius $r_{\max }$ beyond which the plasma density vanishes, the integral is just a constant, $K=1$ and $E_{+} / E_{-}$falls of as $r^{-2 m}$. Equation (26) can be integrated by parts to give

$$
R_{m}(r)=-\frac{2 m}{r^{2 m}} \int_{0}^{r_{\max }}\left[\frac{1}{K\left(r^{\prime}\right)}-1\right] r^{\prime 2 m-1} d r^{\prime},
$$

for $r>r_{\max }$. Using the definition of $K\left(r^{\prime}\right)$ this can also be written

$$
R_{m}(r)=-\frac{2 m}{r^{2 m}} \int_{0}^{r_{\max }}\left[\frac{f\left(r^{\prime}\right)}{\lambda-(m-1) g\left(r^{\prime}\right)}\right] r^{\prime 2 m-1} d r^{\prime} .
$$

Here, $R_{m}$ is a measure of the $m$ th multipole moment induced in the plasma column by the applied field. For the illustrative profile of Fig. 2, $r_{\max }=a$.

\section{ADMITTANCE FUNCTION}

The expression of $R_{m}(r)$ is now related to the measurement geometry depicted in Fig. 1. If a voltage $V e^{-i \omega t}$ is applied to the segmented cylinder shown, the current $I e^{-i \omega t}$ flowing to the electrode (as displacement current) will be

$$
I=-i \omega \epsilon_{0} \int_{-\theta_{0}}^{\theta_{0}} E_{1 r} b d \theta .
$$

It is then straightforward to show that the observed admittance $Y=I / V$ is related to the $R_{m}(b)$ defined above as follows:

$$
Y(\lambda)=-i \omega_{c} \epsilon_{0} b \sum_{m} C_{m} \frac{R_{m}(b)+1}{R_{m}(b)-1},
$$

where the $C_{m}=(4 m / \pi)\left[\sin \left(m \theta_{0}\right) / m\right]^{2}$ are geometrical weighting factors for the various multipole contributions which depend only upon $m$ and the angle $\theta_{0} ; C_{m}$ falls off like $1 / m$ and for $\theta_{0}=\pi / 2$ only the odd $C_{m}$ are nonzero. The fraction $\left(R_{m}+1\right) /\left(R_{m}-1\right)$ is just proportional to the $m$ th multipole component of $E_{1} / E_{1 \theta}$ at the electrode, and from Eq. (29) it is evident that we should examine the behavior of that factor for various $m$. Because of the factor $\left(r^{\prime} / b\right)^{2 m}$ in Eq. (26) the higher multipole contributions fall off as $(a / b)^{2 m}$, where we have set $r_{\max }=a$. Thus the dipole contribution $(m=1)$ will dominate. Subtracting the vacuum contribution to the admittance, i.e., that part which exists in the absence of the plasma, the appropriate factor in Eq. (29) becomes $2 R_{m} /\left(R_{m}-1\right)$.

\section{A. Plasma density independent of radius}

For plasma of uniform density up to radius $a, f=g=1$ for $r<a$ and Eq. (27a) is particularly easy to evaluate and gives

$$
R_{m}(b)=-(a / b)^{2 m} \frac{1}{\lambda-m+1},
$$

so that

$$
\frac{R_{m}(b)+1}{R_{m}(b)-1}=-\frac{\lambda-m+1-(a / b)^{2 m}}{\lambda-m+1+(a / b)^{2 m}} .
$$

Equation (31) has a simple pole at

$$
\lambda=m-1-(a / b)^{2 m}=m-2+1-(a / b)^{2 m},
$$

with a residue $-2(a / b)^{2 m}$. Thus there is a single discrete mode for each $m$, with the coupling decreasing with $m$, in accord with earlier work. ${ }^{3}$ Each term in the second form of Eq. (32) can be identified with a frequency shift of a particular origin. The first is a Doppler shift due to plasma rotation. The second is a downshift of the single particle gyration frequency. The third is a plasma upshift giving rise to the upper hybrid frequency. The last is a downshift caused by the image charges in the conducting walls. For $(b / a)^{2 m} \geqslant 1$ the influence of the walls is negligible and this shift may be neglected. In a neutral plasma, the first two shifts are absent. Note that the $m=1$ resonance lies below the free particle cyclotron frequency and $m=2$ and higher resonances lie above.

\section{B. Plasma density dependent upon radius, $m=1$}

When the equilibrium density, and therefore the rotational angular velocity, described by $f(r)$ and $g(r)$, respectively, depends on radius the situation is more complicated, except for $m=1$. For $m=1$, the radial dependence in the denominator of Eq. (27a) disappears and $R_{1}(b)$ is readily found to be

$$
R_{1}(b)=-\frac{1}{\lambda}\left[\frac{1}{b^{2}} \int_{0}^{b} f(r) 2 r d r\right]=-\frac{\langle f\rangle}{\lambda},
$$

and

$$
\frac{R_{1}+1}{R_{1}-1}=\frac{\lambda-\langle f\rangle}{\lambda+\langle f\rangle},
$$

where $\langle f\rangle$ is the average of $f$ over the entire cross section. Thus the $m=1$ resonance occurs at $\lambda=-\langle f\rangle$, i.e. it is downshifted and the downshift depends solely on the density averaged over the cross section. This downshift is exactly equal to the frequency of the low-frequency $m=1$ diocotron mode. In both the $m=1$ cyclotron mode and the $m=1$ diocotron mode are center of mass modes. The downshift of the cyclotron mode and the frequency of the diocotron mode both arise from image charges in the conducting wall, as evidenced by the fact that as $a / b$ tends to zero (wall far away) both vanish. Wall charges produce a downshift because the force which they cause on the charged particles is antiparallel to the $\mathbf{v} \times \mathbf{B}$ force, thus reducing slightly the frequency of 
circular motion. The sum of the frequencies of the downshifted cyclotron mode and low-frequency diocotron mode is equal to $\omega_{c}=e B_{0} / m_{e}$.

\section{Plasma density dependent upon radius, $m>1$}

The behavior of the $m>1$ modes is not as simple as for $m=1$ when the density depends upon radius. Then the denominator of Eq. (27a) will vanish at at some radius where $\lambda=(m-1) g(r)$. This is just the condition at which the dielectric function $K$ vanishes and therefore $E$. becomes very large. This condition is satisfied only at a particular radius, which depends upon the frequency $\lambda$, so there is a localized resonant layer, the upper-hybrid layer for a non-neutral plasma. Denoting the minimum and maximum normalized rotation frequencies where $f \neq 0$, then an upper-hybrid resonant layer occurs in the frequency band

$$
(m-1) g_{\min }<\lambda<(m-1) g_{\max } \text {. }
$$

One can look at this another way. In the frequency band just described, there exists a continuum of singular normal modes, with singularity occurring at the radius $r_{s}$ where $(m-1) g\left(r_{s}\right)=\lambda$. Based on the previous analysis, a prescription for finding the field of a singular normal modes is as follows. For a particular $m$, pick a frequency $\lambda$ in the proper frequency band. Find $E_{-}(r)$ from Eq. (23) by interpreting $1 / K(r)=P\{1 / K(r)\}+\mu \delta\left(r-r_{s}\right)$ where $P\{\}$ stands for the principal value and $\mu$ is a constant to be determined. Substitute this result into Eq. (25) to find $E_{+}(r)$. Finally, determine the constant $\mu$ by requiring that $2 i E_{\theta}(b)=E_{+}(b)-E_{-}(b)=0$, since $r=b$ is a conducting boundary. Application of this procedure for $\lambda$ 's in the frequency band described by Eq. (24) presumably gives a complete orthogonal set of singular eigenfunctions.

For the evaluation of the $R_{m}$ 's which appear in the admittance function $Y$, Eq. (29) can be regarded as resulting from the analysis of an initial value problem in which $\lambda$ has a positive imaginary part, thus resolving the question of how to handle the singularity at $r=r_{s}$. Alternatively, we could have assumed the existence of a small collision frequency in the earlier analysis and this would have the effect of replacing $\lambda$ by $\lambda+i \nu$. For the purpose of evaluating and displaying the various multipole contributions to the admittance function, Eq. (29), the latter approach is followed, taking the limit of very small $\nu$. For the parabolic profiles previously discussed, the expression for $R_{m}(b)$ can be integrated to give

$$
\begin{aligned}
R_{m}(b)= & -2 m\left(\frac{a}{b}\right)^{2 m}\left[\sum_{k=0}^{m-2} \frac{\Lambda^{k}}{(m-k)(m-k-1)}-\Lambda^{m-1}\right. \\
& \left.-\Lambda^{m-1}(\Lambda-1) \ln \left(\frac{\Lambda-1}{\Lambda}\right)\right],
\end{aligned}
$$

where $\Lambda=-2(\lambda-m+1) /(m-1)$. When $0<\Lambda<1$, the logarithm is complex and this leads to a complex admittance function representing absorption associated with a resonant layer in the plasma. The frequency band in which this occurs is just that given by Eq. (34) with $g_{\min }=0.5$ and $g_{\max }=1$. In particular for $m=2$,

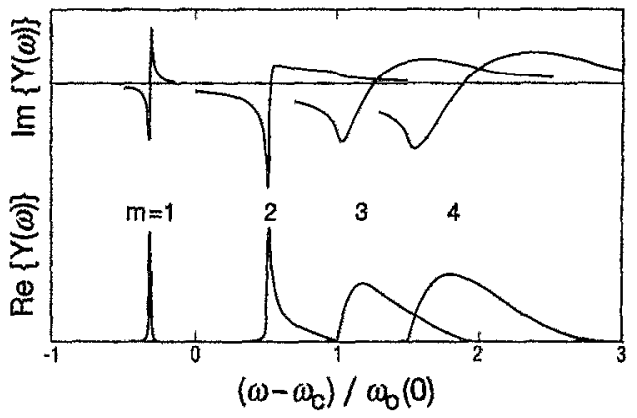

FIG. 3. Admittance versus frequency for the $m=1,2,3,4$ modes and the profiles shown in Fig. 2. Plotted are the real and imaginary parts of the plasma contribution, $2 R_{m}(b) /\left[R_{m}(b)-1\right]$. The ratio of wall radius, $b$, to plasma radius. $a$ is 1.25. Plots for $m=3$ and 4 have been expanded by factors of 5 and 10 with respect to $m=2$ for clarity in presentation.

$$
\begin{aligned}
R_{2}(b)= & -2\left(\frac{a}{b}\right)^{4}[(4 \lambda-3)-4(1-\lambda) \\
& \left.\times(2 \lambda-1) \ln \left(\frac{1-2 \lambda}{2(1-\lambda)}\right)\right] .
\end{aligned}
$$

In Fig. 3 the real and imaginary parts of $2 R_{m}(b) /\left[R_{m}(b)-1\right]$, the main factor in the admittance $Y_{\text {, }}$ is displayed as a function of $\lambda$. This clearly displays both the absorption band in which $Y$ has a real part, and the existence of a localized upper hybrid resonance layer and the dependence of its position on $\lambda$.

\section{RESONANCES OF THE WARM PLASMA}

The previous discussion assumed zero temperature electrons and this led to singular electric fields at the radius where $K(r)$ vanishes. Under these conditions, finite Larmor radius effects (FLR) are important and lead to significant modifications of the behavior described above. Singular eigenmodes are no longer possible and Bernstein modes, propagating across the magnetic field, become possible. An approximate treatment of consequences of FLR effects is now developed by modifying the diclectric function $K(r)$ obtained earlier to include FLR effects, to first order in the plasma temperature. When the disturbance takes the form of a plane wave with wave number $k$, the result of FLR effects is to reduce the effectiveness of the electric field in producing plasma currents by a factor $4 I_{1}\left(k^{2} \rho^{2} / 2\right)$ $\cdot e^{-\left(k^{2} \rho^{2} / 2\right) /(k \rho)^{2}}$, where $I_{1}$ is the modified Bessel function and $\rho^{2}$ is the mean square Larmor radius, averaged over a Maxwellian distribution. To first order in $(k \rho)^{2}$, i.e., to first order in the temperature, this factor is approximately $1-0.5(k \rho)^{2}$. This approximation is useful so long as the Larmor radius is small compared with the wavelength. Since the particles average the electric field over the orbit, the dielectric function is no longer local. Neighboring regions of the plasma are coupled together and the continuum of singular normal modes is replaced by a discrete set of Bernstein modes. This result is now applied to a situation where the waves are not strictly plane waves because the plasma properties vary slowly with position. This approximation is useful so long as the wavelength of the wave is small compared 
with the distance over which the plasma properties vary. This leads to the following modification of the dielectric function:

$$
K(r)=1-\frac{f\left[1-0.5(k \rho)^{2}\right]}{\lambda-m g+g+f},
$$

where $f$ and $g$ have their previous meanings and are functions of radius. However, it should be pointed out that while temperature has been included to first order in the form of FLR effects in the wave dynamics, the effect of temperature in the form of the diamagnetic contribution to the angular velocity has not been included. This will modify slightly the relationship between $f$ and $g$. As before, Eq. (36) is valid only around the cyclotron frequency. This result is now applied to the nonplane wave fields replacing $k^{2}$ by $-\nabla^{2}$, the Laplace operator. This assumes that the density and angular velocity scale lengths are large compared to the wavelength of the waves. This leads to an approximate wave equation for the Bernstein modes which will determine the eigenmodes of the system,

$$
\frac{1}{r} \frac{d}{d r}(r R)-\frac{m^{2}}{r^{2}} R+k^{2}(r) R=0,
$$

where

$$
k^{2}(r)=\frac{2[(m-1) g(r)-\lambda]}{\rho^{2} f(r)} ;
$$

$k(r)$ could be interpreted as the local wave number and it obviously depends upon radius through $g(r)$ and $f(r)$. When $k^{2}(r)>0$ the waves are propagating and when $k^{2}(r)<0$ the waves are evanescent, with $k=0$ defining the turning point.

To proceed further, an assumption about the density profile function $f(r)$ must be made. According to Eq. $\left(8^{\prime}\right)$ this also determines the angular velocity profile function $g(r)$. For simplicity the parabolic density and angular velocity profiles illustrated in Fig. 2 are used. This choice of $f(r)$ is very close to a diffusion profile, $f(r)=J_{0}(2.405 r / a)$ if $a=b$, the radius of the wall. For $(m-1) g(r)>\lambda$ the Bernstein waves are propagating and for $(m-1) g(r)<\lambda$ the waves are evanescent. For $m>1$ and $\lambda$ a little less than $(m-1)$ the waves are trapped in the inner part of the plasma coliumn and evanescent in the outer part of the column (like the Buchsbaum-Hasegawa modes ${ }^{9}$ near $2 \omega_{c}$ and opposite from the Tonks-Dattner modes ${ }^{13}$ near $\omega_{p}$, which are trapped in the outer region of the plasma. The lowest modes are trapped quite close to the center where $f(r) \approx 1$. If this approximation is made in Eq. (38) then Eq. (37) becomes the twodimensional harmonic oscillator equation, whose eigenvalues are readily shown to be

$$
\lambda_{l m}=(m-1)-\sqrt{m-1}(2 l+m-1) \frac{\rho}{a},
$$

where $l$ is the radial mode number.

Alternatively, Eq. (37) can be integrated numerically to obtain the eigenvalues. The appropriate boundary conditions are $E_{-} \sim r^{m-1}$ near the origin and $E_{-}=0$ at $r=a$ [one solution of Eq. (37) can be shown to have such behavior at $r=a]$. One begins the integration at $r=0$ and adjusts the eigenvalue $\lambda$ in successive integrations so as to assure the

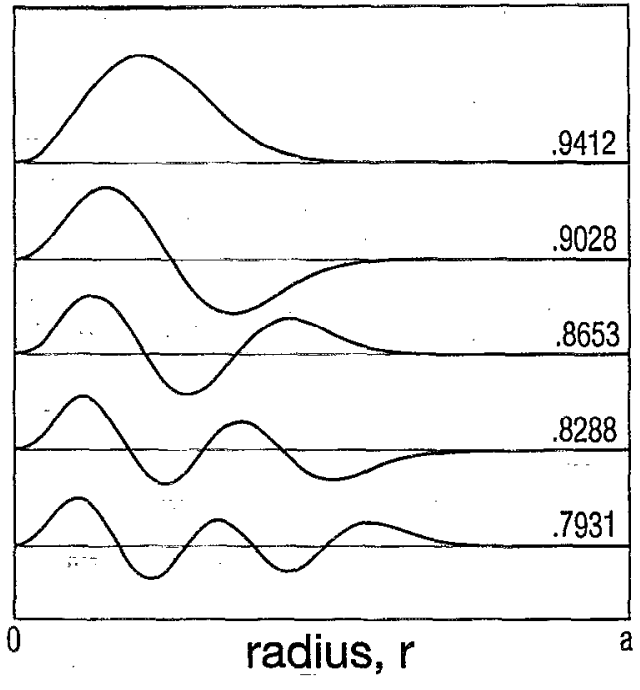

FIG. 4. Radial wave functions for $m=2, \rho / a=0.02$, with their normalized frequencies (from Ref. 1). The first five radial modes are shown.

eigenfunction vanishes at $r=a$. The radial dependences of the first few $m=2$ modes obtained in this manner are shown in Fig. 4. One can see from Fig. 4 that the turning point for successively higher radial trapped Bernstein modes is closer to the plasma edge and would, for higher radial mode numbers, approach the wall. Under these circumstances, the evanescent layer becomes thin and one can no longer argue that the conditions at the edge of the plasma are unimportant. Thus this approximate approach breaks down for very high radial mode numbers. The eigenvalues obtained are plotted in Fig. 5 for the range $0<\rho / a<0.05$. For very small values of this parameter (low temperature) the Bernstein mode spectrum is very dense, approaching a continuous spectrum, as found earlier, when $T \rightarrow 0$. For a given $m>0$, the eigenvalues all have $\lambda<m-1$, and for this profile $\lambda>(m-1) / 2$.

\section{EXTENSION TO MULTIPLE ION SPECIES}

Only a few changes are required to extend the previous results to two or more ion species. This extension is motivated by the recent experiments of Sarid et al. ${ }^{14}$ First, the discussion is limited to two species for simplicity: Second, because the particlès have positive charge, they gyrate in the opposite direction, the plasma rotation is in the opposite direction, and fields which can drive cyclotron resonance have the opposite polarization. In the discussion below, we simply ignore these changes in sign and extend the previous analysis for electrons of different mass, and make appropriate changes. Thus the quantities $\omega_{c}$ and $\omega_{0}$ below are positive. As before the density profiles of the two species are characterized by ::....

$$
\begin{aligned}
& n_{01}(r)=n_{01}(0) f_{\mathrm{i}}(r), \quad f_{1}(0)=1 \\
& n_{02}(r)=n_{02}(0) f_{2}(r), \quad f_{2}(0)=\mathrm{I} .
\end{aligned}
$$

The $\mathbf{E} \times \mathbf{B}$ angular velocity of each species is the same so a common angular velocity function $g(r)$ is used, 


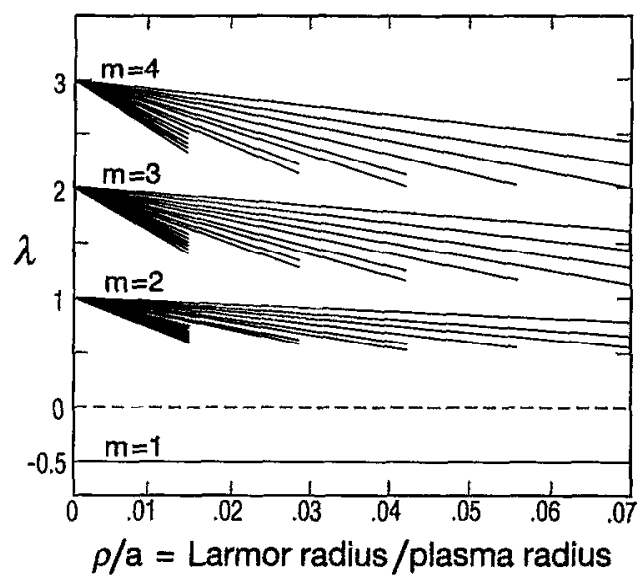

FIG. 5. Theoretical spacing of the trapped $m=2$ Bernstein modes versus Larmor radius, $\rho / a$, normalized to the central rotation frequency $f_{p}^{2}(0) / 2 f_{c}$ for the profiles of Fig. 2 (from Ref. 1).

$$
\omega_{0}(r)=\omega_{0}(0) g(r), \quad g(0)=1,
$$

where

$$
\omega_{0}(0)=\frac{\omega_{p 1}^{2}(0)}{2 \omega_{c 1}}+\frac{\omega_{p 2}^{2}(0)}{2 \omega_{c 2}} .
$$

Let $\epsilon_{2}=\omega_{p 2}^{2} / 2 \omega_{c 2} /\left(\omega_{p 2}^{2} / 2 \omega_{c 2}+\omega_{p 1}^{2} / 2 \omega_{c 1}\right)$ be the charge fraction of species 2 (with a similar definition for $\epsilon_{1}$ ). Then Eq. $\left(8^{\prime}\right)$ becomes

$$
\frac{1}{r} \frac{d}{d r}\left[r^{2} g(r)\right]=2\left[\epsilon_{1} f_{1}(r)+\epsilon_{2} f_{2}(r)\right] .
$$

When $f_{1}(r)$ and $f_{2}(r)$ are specified $g(r)$ is determined. For example, if $f_{1}(r)=1-\alpha_{1}(r / a)^{2}$ and $f_{2}(r)=1-\alpha_{2}(r / a /)^{2}$ then

$$
g(r)=1-\frac{\alpha_{1} \epsilon_{1}+\alpha_{2} \epsilon_{2}}{2}\left(\frac{r}{a}\right)^{2} .
$$

Parabolic profiles serve as simple illustrative examples of diffusion-like nonrigid rotor profiles, and reduce to $T=0$ rigid rotor profiles when the $\alpha$ 's are set to zero.

We now examine cyclotron resonance when the frequency is near the cyclotron frequency of species 1 . The dynamics of species 2 can be ignored, since it is far from resonance. However, its effect on $\omega_{0}(r)$ has been included in $g(r)$. As before, a frequency shift, $\lambda_{1}$, normalized to the central rotation frequency, is introduced for the mode near $\omega \approx \omega_{c l}$,

$$
\omega=\omega_{c 1}+\omega_{0}(0) \lambda_{1} .
$$

The analysis leading up to Eq. (19) is unchanged, except that $f(r)$ is replaced by $\epsilon_{1} f_{1}(r)$. The dielectric function for circularly polarized fields becomes

$$
K(r)=1-\frac{\epsilon_{1} f_{1}(r)}{\lambda_{1}-(m-1) g(r)-0.5 r d g(r) / d r},
$$

and when Eq. (43) is used to solve for $1 / K-1$ we obtain

$$
\frac{1}{K(r)}-1=\frac{\epsilon_{1} f_{1}}{\lambda_{1}-(m-1) g+\epsilon_{2} f_{2}},
$$

with a similar expression (with subscripts 1 and 2 interchanged) when $\omega \approx \omega_{\mathrm{c} 2}$. For the plasma extending to $r=a$ and the wall at $r=b$ we get

$$
R_{m}(b)=-\frac{1}{b^{2 m}} \int_{0}^{a} \frac{\epsilon_{1} f_{1}}{\lambda_{1}-(m-1) g+\epsilon_{2} f_{2}} 2 m r^{2 m-1} d r .
$$

This gives the behavior when the frequency is in the vicinity of $\omega_{c 1}$ and a similar expression (with the subscripts 1 and 2 interchanged) gives the behavior when the frequency is in the vicinity of $\omega_{c 2}$. When $\epsilon_{2}=0$ (no second species) and $m=1$, Eq. (33), our previous single species result, is recovered. As in the single species case, the two-species integrals such as Eq. (47) can be evaluated in the case of a parabolic density profile, and this evaluation leads to logarithmic functions as given in Eq. (35).

\section{A. Plasma density independent of radius}

For rigid rotor, or "top hat" profiles, $f_{1}(r)=f_{2}(r)=g(r)=1$ for $r \leqslant a$ so that

$$
\begin{aligned}
& R_{m}(b)=-\frac{\epsilon_{1}}{\lambda_{1}-(m-1)+\epsilon_{2}}\left(\frac{a}{b}\right)^{2 m}, \quad \omega \approx \omega_{c 1}, \\
& R_{m}(b)=-\frac{\epsilon_{2}}{\lambda_{2}-(m-1)+\epsilon_{1}}\left(\frac{a}{b}\right)^{2 m}, \quad \omega \approx \omega_{c 2} .
\end{aligned}
$$

The resonant modes are obtained by setting $R_{m}(b)$ equal to unity and this gives

$$
\begin{aligned}
& \lambda_{1}=(m-1)-\epsilon_{2}-\frac{a^{2 m}}{b^{2 m}} \epsilon_{1}, \quad \omega \approx \omega_{c 1}, \\
& \lambda_{2}=(m-1)-\epsilon_{1}-\frac{a^{2 m}}{b^{2 m}} \epsilon_{2}, \quad \omega \approx \omega_{c 2} .
\end{aligned}
$$

Upon comparison with Eq. (32) it is seen that the terms involving $\epsilon$ describe the changes in resonant frequencies arising from the presence of $a$ another species. For the center of mass modes $(m=1)$ :

$$
\begin{aligned}
& \lambda_{1}=-\frac{a^{2}}{b^{2}}-\left(1-\frac{a^{2}}{b^{2}}\right) \epsilon_{2}, \\
& \lambda_{2}=-\frac{a^{2}}{b^{2}}-\left(1-\frac{a^{2}}{b^{2}}\right) \epsilon_{1},
\end{aligned}
$$

where the fact that $\epsilon_{1}+\epsilon_{2}=1$ has been used. The second term, proportional to the density of the other species, represents an additional downshift caused by the presence of the other species.

\section{B. Plasma density dependent upon radius}

We now consider density profile effects and, for simplicity, limit our discussion to the parabolic profiles used earlier. From Eq. (47), and the similar expression with subscripts 1 and 2 interchanged, we note that it is possible for the denominator of the integral to vanish at some radius in certain 
frequency bands. In the cold plasma theory this signifies absorption bands and in the warm plasma theory these are the bands in which the Bernstein modes may be found. To determine these frequency bands consider the vanishing of the denominator with the assumed profiles. Note that bands associated with each of the species is expected. The denominator for the species 1 resonance has the form

$$
\lambda_{1}-(m-1)\left(1-\frac{\alpha}{2} \frac{r^{2}}{a^{2}}\right)+\epsilon_{2}\left(1-\alpha \frac{r^{2}}{a^{2}}\right),
$$

where, for simplicity, it has been assumed that $\alpha_{1}=\alpha_{2}=\alpha$, i.e., that both species have the same density profile. Vanishing of this denominator at $r=0$ and $r=a$ serves to determine the limits of the absorption bands. These limits are, for species 1 ,

$$
\begin{aligned}
& \lambda_{1}=(m-1)-\epsilon_{2}, \\
& \lambda_{1}=(m-1)\left(1-\frac{\alpha}{2}\right)-\epsilon_{2}(1-\alpha),
\end{aligned}
$$

with similar expressions for the limits associated with the second species. For the center of mass modes $(m=1)$, the frequencies of these two bañds can be described as

$$
\begin{aligned}
& -\epsilon_{2} \leqslant \lambda_{1} \leqslant-\epsilon_{2}(1-\alpha), \\
& -\epsilon_{1} \leqslant \lambda_{2} \leqslant-\epsilon_{1}(1-\alpha),
\end{aligned}
$$

i.e., both bands lie below their respective cyclotron frequencies. From this result, it is seen that, unless the density is independent of radius ( $\alpha=0$, top-hat distributions), there are now absorption bands associated with the $m=1$ cyclotron modes, whereas for the single species case, there was only a single discrete resonance. We speculate that the inclusion of temperature may lead to Bernstein modes in these bands.

\section{SUMMARY AND CONCLUSIONS}

We have studied the $k_{z}=0$ cyclotron modes of a cylindrical non-neutral plasma of low density $\left(\omega_{p}^{2} / \omega_{c}^{2} \ll 1\right)$. Particular attention has been given to a single species (electrons) which is in nonuniform rotation, i.e., where the density and angular velocity are functions of radius. The general features of the behavior near cyclotron resonance have been studied for a simple parabolic profile. The qualitative behavior for other monotonically decreasing profiles is similar but differs in details. We have obtained admittance function, $Y(\omega)$, which characterizes the current flowing to a segment of the wall when an oscillating potential whose frequency is near the cyclotron frequency is applied to that segment. An absorption band is found for each $m$ value, for the range of frequencies for which a local upper-hybrid resonance occurs at some radius in the nonuniform plasma. These absorption bands correspond the the frequency bands in which the plasma has a continuum of singular eigenfunctions, although that approach is not pursued here. The plasma responds only to the circularly polarized component of the electric field which has the same sense as the gyrating charged particles and, because of plasma rotation, the each absorption band is upshifted by the Doppler effect. Found to be a special case is $m=1$ with only a single discrete mode and no continuum, a property which it shares with the low-frequency $m=1$ diocotron mode. Both are center of mass modes. Presumably in both cases an $m=1$ continuum of singular eigenmodes exists but they have no electric field exterior to the plasma, and therefore not observable, or excitable, with electrodes exterior to the plasma. The $m=1$ cyclotron mode was shown to have a frequency which lies below the cyclotron frequency by and amount equal to the low-frequency diocotron mode frequency $\left\langle\omega_{p}^{2}\right\rangle / 2 \omega_{c}$, where the average is taken over the cross section of the cylinder.

When finite Larmor radius effects, owing to plasma temperature, are taken into account in an approximate fashion, Bernstein modes are found in the frequency bands for which the cold plasma theory predicts an absorption continuum associated with the continuous spectrum of singular eigenmodes. Because of the radial density and angular velocity profiles the Bernstein modes are trapped in the central core of the plasma. They are traveling waves in the azimuthal direction and standing waves in the radial direction. The relative frequency spacing between modes with different numbers of radial nodes is proportional to the ratio of Larmor radius to gradient scale length, a small quantity. The Bernstein mode spacing can be used to estimate the plasma temperature. Finally, an extension of the cold plasma results to plasmas with multiple ion species has been outlined.

A more complete theory of the trapped Bernstein modes in a cylindrical nonuniform nonneutral plasma, such as a solution of the Vlasov equation to first order in the temperature, is desirable. This approach has been used in Ref. 6 for a neutral plasma and in Ref. 8 for a planar non-neutral plasma. In order to carry out such an analysis one would also have to find a suitable steady state with radial profiles of the density and angular velocity which includes the diamagnetic drifts as well as the $E \times B$ drifts.

\section{ACKNOWLEDGMENTS}

The author is pleased to acknowledge E. Sarid for discussion of his experimental results and the Office of Naval Research for support of this work under Grant No. N00001489-J-1264.

'R. W. Gould and M. A. LaPointe, Phys. Rev. Lett. 67, 3685 (1991); Phys. Fluids B 4, 2038 (1992).

${ }^{2}$ Non-Neutral Plasma Physics, AIP Conf. Proc. 175, edited by C. W. Roberson and C. F. Driscoll (American Institute of Physics; New York, 1988)

${ }^{3}$ R. C. Davidson, Physics of Nonneutral Plasmas (Addison-Wesley, Redwood City, CA, 1990).

${ }^{4}$ R. C. Davidson and N. A. Krall, Phys. Rev. Lett. 22, 833 (1969); Phys. Fluids 13, 1543 (1970).

${ }^{5}$ D. H. E. Dubin, Phys. Rev. Lett. 66, 2076 (1991).

${ }^{6} \mathrm{G}$. A. Pearson, Phys. Fluids 9, 2454 (1966).

${ }^{7}$ I. B. Bernstein, Phys. Rev. 10, 109 (1958).

${ }^{8}$ S. A. Prasad, G. J. Morales, and B. D. Fried, Phys. Fluids 30, 3093 (1987).

${ }^{9}$ S. J. Buchsbaum and A. Hasegawa, Phys. Rev. Lett. 12, 685 (1964); Phys. Rev. 143, 303 (1966).

${ }^{10}$ For a good review of Bernstein waves in neutral plasmas, see G. Bekekfi, Radiation Processes in Plasmas (Wiley, New York, 1966).

${ }^{11}$ N. G. Van Kampen, Physica 21, 949 (1955).

${ }^{12}$ K. M. Case; Ann. Phys. 7, 349 (1959).

${ }^{13}$ J. V. Parker, J. C. Nickel, and R. W. Gould, Phys. Rev. Lett. 11, 183 (1963); Phys. Fluids 7, 1489 (1964).

${ }^{14}$ E. Sarid, F. Anderegg, and C. F. Driscoll, Bull. Am. Phys. Soc. 38, 1971 (1993). 